\title{
ON THE NUMBER OF ZEROS OF NONOSCILLATORY SOLUTIONS TO HALF-LINEAR ORDINARY DIFFERENTIAL EQUATIONS INVOLVING A PARAMETER
}

\author{
KUSANO TAKAŜI AND MANABU NAITO
}

\begin{abstract}
In this paper the following half-linear ordinary differential equation is considered:

$\left(\mathrm{H}_{\lambda}\right) \quad\left(\left|x^{\prime}\right|^{\alpha} \operatorname{sgn} x^{\prime}\right)^{\prime}+\lambda p(t)|x|^{\alpha} \operatorname{sgn} x=0, \quad t \geq a$,

where $\alpha>0$ is a constant, $\lambda>0$ is a parameter, and $p(t)$ is a continuous function on $[a, \infty), a>0$, and $p(t)>0$ for $t \in[a, \infty)$. The main purpose is to show that precise information about the number of zeros can be drawn for some special type of solutions $x(t ; \lambda)$ of $\left(\mathrm{H}_{\lambda}\right)$ such that

$$
\lim _{t \rightarrow \infty} \frac{x(t ; \lambda)}{\sqrt{t}}=0 \text {. }
$$

It is shown that, if $\alpha \geq 1$ and if $\left(\mathrm{H}_{\lambda}\right)$ is strongly nonoscillatory, then there exists a sequence $\left\{\lambda_{n}\right\}_{n=1}^{\infty}$ such that $0=\lambda_{0}<\lambda_{1}<\cdots<\lambda_{n}<\cdots, \lambda_{n} \rightarrow+\infty$ as $n \rightarrow \infty$; and $x(t ; \lambda)$ with $\lambda=\lambda_{n}$ has exactly $n-1$ zeros in the interval $(a, \infty)$ and $x\left(a ; \lambda_{n}\right)=0$; and $x(t ; \lambda)$ with $\lambda \in\left(\lambda_{n-1}, \lambda_{n}\right)$ has exactly $n-1$ zeros in $(a, \infty)$ and $x\left(a ; \lambda_{n}\right) \neq 0$. For the proof of the theorem, we make use of the generalized Prüfer transformation, which consists of the generalized sine and cosine functions.
\end{abstract}

\section{INTRODUCTION}

First let us consider second-order half-linear differential equations of the form

$$
\left(\left|x^{\prime}\right|^{\alpha} \operatorname{sgn} x^{\prime}\right)^{\prime}+q(t)|x|^{\alpha} \operatorname{sgn} x=0, \quad t \geq a,
$$

where $\alpha>0$ is a constant and $q(t)$ is a continuous function on $[a, \infty)$. This equation has the following remarkable property: if $x(t)$ is a solution of $(1.1)$, then $c x(t)$ is also a solution of (1.1), where $c$ is any constant. If $\alpha=1$, then equation (1.1) becomes the linear equation

$$
x^{\prime \prime}+q(t) x=0, \quad t \geq a .
$$

If $\alpha \neq 1$, then (1.1) is no longer linear. However, the half-linear equation (1.1) has essentially the same properties as the linear equation (1.2). It is known ([16) that for any initial condition $x(b)=x_{0} \in \boldsymbol{R}, x^{\prime}(b)=x_{1} \in \boldsymbol{R}(b \geq a)$, equation (1.1) has a unique solution $x(t)$ on the interval $[a, \infty)$. Therefore, a nontrivial solution $x(t)$ of (1.1) has either a finite number of zeros on $[a, \infty)$, in which case $x(t)$ is called nonoscillatory, or an infinite number of zeros clustering at $t=\infty$,

Received by the editors January 5, 2001

2000 Mathematics Subject Classification. Primary 34C10; Secondary $34 \mathrm{~B} 16$.

Key words and phrases. Half-linear equations, zeros of nonoscillatory solutions. 
in which case $x(t)$ is called oscillatory. Furthermore, Sturmian separation and comparison theorems can be established $([4,15,16])$ for the half-linear equation (1.1) as a natural extension of (1.2). Thus nontrivial solutions of (1.1) are either all nonoscillatory or else all oscillatory. As usual, if the former occurs, then (1.1) is called nonoscillatory, and if the latter occurs, then (1.1) is called oscillatory.

In this paper we consider the following half-linear differential equation with a parameter:

$$
\left(\left|x^{\prime}\right|^{\alpha} \operatorname{sgn} x^{\prime}\right)^{\prime}+\lambda p(t)|x|^{\alpha} \operatorname{sgn} x=0, \quad t \geq a,
$$

under the hypotheses that

(a) $\alpha>0$ is a constant;

(b) $\lambda>0$ is a parameter;

(c) $p(t)$ is a continuous function on $[a, \infty), a>0$, and $p(t)>0$ for $t \in[a, \infty)$.

As in the linear case, we say that $\left(\mathrm{H}_{\lambda}\right)$ is strongly nonoscillatory [resp. strongly oscillatory] if $\left(\mathrm{H}_{\lambda}\right)$ is nonoscillatory [resp. oscillatory] for every $\lambda>0$. A complete characterization of strong nonoscillation and strong oscillation is obtained in the following theorem, which is a direct generalization of a result of Nehari 17 .

Theorem A (Kusano, Y. Naito and Ogata [13]).

(i) $\left(\mathrm{H}_{\lambda}\right)$ is strongly nonoscillatory if and only if

$$
\int_{a}^{\infty} p(s) d s<+\infty \quad \text { and } \quad \lim _{t \rightarrow \infty} t^{\alpha} \int_{t}^{\infty} p(s) d s=0 .
$$

(ii) $\left(\mathrm{H}_{\lambda}\right)$ is strongly oscillatory if and only if

$$
\int_{a}^{\infty} p(s) d s=+\infty \quad \text { or } \quad \limsup _{t \rightarrow \infty} t^{\alpha} \int_{t}^{\infty} p(s) d s=+\infty .
$$

In this paper we are interested in the situation where $\left(\mathrm{H}_{\lambda}\right)$ is strongly nonoscillatory and are concerned with the problem of counting the number of zeros of (nonoscillatory) solutions of $\left(\mathrm{H}_{\lambda}\right)$. The main purpose of this paper is to show that, in the case $\alpha \geq 1$, precise information about the number of zeros can be drawn for some special type of solutions $x_{\lambda}(t)$ of $\left(\mathrm{H}_{\lambda}\right)$ such that

$$
\lim _{t \rightarrow \infty} \frac{x_{\lambda}(t)}{\sqrt{t}}=0 .
$$

The existence and the essential uniqueness of a nonoscillatory solution $x_{\lambda}(t)$ that satisfies (1.5) are guaranteed by the next theorem.

Theorem 1.1. Let $\alpha>0$ and suppose that (1.3) holds. Then, for each $\lambda>0$, there is a nonoscillatory solution $x_{\lambda}(t)$ of $\left(\mathrm{H}_{\lambda}\right)$ satisfying (1.5). Furthermore, if $\alpha \geq 1$, then such a solution $x_{\lambda}(t)$ is uniquely determined up to a nonzero constant multiple.

For the solutions $x_{\lambda}(t)$ obtained in Theorem 1.1, we can establish the following theorem concerning the number of zeros.

Theorem 1.2. Let $\alpha \geq 1$ and suppose that (1.3) holds. Then there exists a sequence $\left\{\lambda_{n}\right\}_{n=1}^{\infty}$ of positive parameters with the properties that

(i) $0=\lambda_{0}<\lambda_{1}<\cdots<\lambda_{n}<\cdots, \lim _{n \rightarrow \infty} \lambda_{n}=+\infty$;

(ii) if $\lambda \in\left(\lambda_{n-1}, \lambda_{n}\right), n=1,2, \cdots$, then $x_{\lambda}(t)$ has exactly $n-1$ zeros in the open interval $(a, \infty)$ and $x_{\lambda}(a) \neq 0$; 
(iii) if $\lambda=\lambda_{n}, n=1,2, \cdots$, then $x_{\lambda}(t)$ has exactly $n-1$ zeros in the open interval $(a, \infty)$ and $x_{\lambda}(a)=0$.

Theorem 1.2 for the case $\alpha=1$ is given in [9], and Theorem 1.2 is closely related to the results in [6]. It has been shown in [6] that if $\alpha>0$ and

$$
\int_{a}^{\infty} p(s) d s<+\infty \text { and } \int_{a}^{\infty}\left(\int_{s}^{\infty} p(r) d r\right)^{1 / \alpha} d s<+\infty
$$

then equation $\left(\mathrm{H}_{\lambda}\right)$ has a unique solution $x_{\lambda}(t)$ satisfying

$$
\lim _{t \rightarrow \infty} x_{\lambda}(t)=1 \text {, }
$$

and there exists a sequence $\left\{\lambda_{n}\right\}_{n=1}^{\infty}$ having the same properties as in Theorem 1.2. We find that if (1.6) is satisfied, then (1.3) holds. Indeed, this is easily observed from the following fact:

$$
\begin{aligned}
0<\frac{t}{2}\left(\int_{t}^{\infty} p(s) d s\right)^{1 / \alpha} & \leq \int_{t / 2}^{t}\left(\int_{s}^{\infty} p(r) d r\right)^{1 / \alpha} d s \\
& \leq \int_{t / 2}^{\infty}\left(\int_{s}^{\infty} p(r) d r\right)^{1 / \alpha} d s \rightarrow 0 \text { as } t \rightarrow \infty
\end{aligned}
$$

Further, it is clear that (1.7) implies (1.5). Thus we can say that Theorem 1.2 gives a partial extension of the previous result in [6]. Note that Theorem 1.2 is considered for the case $\alpha \geq 1$. The case $0<\alpha<1$ is presently open. It is worth pointing out that (1.3) is the best condition to obtain the conclusion of Theorem 1.2, because (1.3) is a necessary and sufficient condition for the strong nonoscillation of $\left(\mathrm{H}_{\lambda}\right)$.

Under the condition

$$
\int_{a}^{\infty} s^{\alpha+1} p(s) d s<+\infty
$$

an analogous theorem can be proved $\left([\underline{6]})\right.$ for the solutions $x_{\lambda}(t)$ of $\left(\mathrm{H}_{\lambda}\right)$ such that

$$
\lim _{t \rightarrow \infty}\left(x_{\lambda}(t)-t\right)=0 .
$$

However, the establishment of the corresponding result under the weaker condition (1.3) seems to be difficult.

By Theorem 1.2 we have the following corollary, which is concerned with the singular eigenvalue problem

$$
\left\{\begin{array}{l}
\left(\left|x^{\prime}\right|^{\alpha} \operatorname{sgn} x^{\prime}\right)^{\prime}+\lambda p(t)|x|^{\alpha} \operatorname{sgn} x=0, \quad t \geq a \\
x(a)=0 \text { and } \lim _{t \rightarrow \infty} \frac{x(t)}{\sqrt{t}}=0
\end{array}\right.
$$

Corollary 1.1. Let $\alpha \geq 1$ and suppose that (1.3) holds. Then the totality of eigenvalues of the singular eigenvalue problem (1.8) is written as a sequence $\left\{\lambda_{n}\right\}_{n=1}^{\infty}$, where $0<\lambda_{1}<\cdots<\lambda_{n}<\cdots, \lim _{n \rightarrow \infty} \lambda_{n}=+\infty$, and the eigenfunction $x_{\lambda_{n}}(t)$ of (1.8) associated with $\lambda=\lambda_{n}$ has exactly $n-1$ zeros on $(a, \infty)$.

Qualitative properties of solutions to the half-linear equation (1.1) were first discussed by Mirzov [16] and Elbert [4], who showed in particular that the Sturm comparison and separation theorems for the linear equation (1.2) could be extended in a natural way to the half-linear equation (1.1). Further analysis on the oscillatory and nonoscillatory properties of solutions of (1.1) has been made by several authors 
(see, e.g., 3, 15, 8, 12, 13, 14, 15]). Their study shows that most of the basic results for the linear equation (1.2) can be completely extended to the half-linear equation (1.1).

The results for linear eigenvalue problems can also be generalized to half-linear equations. For the regular eigenvalue problem on the compact interval $[a, b]$ with the boundary conditions

$$
A x(a)-A^{\prime} x^{\prime}(a)=0, \quad B x(b)+B^{\prime} x^{\prime}(b)=0,
$$

the reader is referred to the papers $[4,10,11]$. As mentioned above, the singular problem on the infinite interval $[a, \infty)$ with the boundary conditions

$$
x(a)=0, \quad \lim _{t \rightarrow \infty}(x(t)-1)=0
$$

and

$$
x(a)=0, \quad \lim _{t \rightarrow \infty}(x(t)-t)=0
$$

has been discussed in the paper [6].

\section{Preparatory Results}

In this section we will consider the half-linear equation without a parameter

$$
\left(\left|x^{\prime}\right|^{\alpha} \operatorname{sgn} x^{\prime}\right)^{\prime}+p(t)|x|^{\alpha} \operatorname{sgn} x=0, \quad t \geq a,
$$

where $\alpha$ and $p(t)$ are assumed to satisfy the same conditions as $\left(\mathrm{H}_{\lambda}\right)$, that is, $\alpha>0$ and $p(t)$ is continuous on $[a, \infty), a>0$, and $p(t)>0$ for $t \in[a, \infty)$. The results of this section are effectively used for the proofs of Theorems 1.1 and 1.2.

First we prove the following lemma.

Lemma 2.1. Let $\alpha>0$ and suppose that $p(t)$ is continuous on $[a, \infty), a>0$, and $p(t)>0$ for $t \in[a, \infty)$. Then, condition (1.3) is satisfied if and only if

$$
\int_{a}^{\infty} s^{\alpha / 2} p(s) d s<+\infty \text { and } \lim _{t \rightarrow \infty} t^{\alpha / 2} \int_{t}^{\infty} s^{\alpha / 2} p(s) d s=0 .
$$

Proof. Suppose that (2.2) is satisfied. Then, noting that

$$
0<t^{\alpha} \int_{t}^{\infty} p(s) d s \leq t^{\alpha / 2} \int_{t}^{\infty} s^{\alpha / 2} p(s) d s \rightarrow 0 \quad \text { as } t \rightarrow \infty,
$$

we have (1.3). Conversely, let (1.3) hold. For simplicity we put

$$
F(t)=\int_{t}^{\infty} p(s) d s, \quad t \geq a .
$$

Then it is easy to see that

$$
\int_{t}^{\tau} s^{\alpha / 2} p(s) d s=-\tau^{\alpha / 2} F(\tau)+t^{\alpha / 2} F(t)+\frac{\alpha}{2} \int_{t}^{\tau} s^{(\alpha / 2)-1} F(s) d s
$$

for $\tau>t$. By (1.3) we have $F(t) \leq t^{-\alpha}$ for all large $t$; hence

$$
\int_{t}^{\tau} s^{(\alpha / 2)-1} F(s) d s \leq \int_{t}^{\tau} s^{-(\alpha / 2)-1} d s \leq \int_{t}^{\infty} s^{-(\alpha / 2)-1} d s<+\infty
$$

for all large $t$. This implies

$$
\int_{t}^{\infty} s^{(\alpha / 2)-1} F(s) d s<+\infty .
$$


Noting that $\lim _{\tau \rightarrow \infty} \tau^{\alpha / 2} F(\tau)=0$ and taking the limit as $\tau \rightarrow \infty$ in (2.3), we have

$$
\int_{t}^{\infty} s^{\alpha / 2} p(s) d s=t^{\alpha / 2} F(t)+\frac{\alpha}{2} \int_{t}^{\infty} s^{(\alpha / 2)-1} F(s) d s,
$$

which gives

$$
t^{\alpha / 2} \int_{t}^{\infty} s^{\alpha / 2} p(s) d s=t^{\alpha} F(t)+\frac{\alpha}{2} t^{\alpha / 2} \int_{t}^{\infty} s^{(\alpha / 2)-1} F(s) d s .
$$

The first term of the right-hand side of (2.4) tends to 0 as $t \rightarrow \infty$. For the second term of the right-hand side of (2.4), rewriting this term as

$$
\frac{\int_{t}^{\infty} s^{(\alpha / 2)-1} F(s) d s}{t^{-(\alpha / 2)}},
$$

and applying l'Hospital's rule, we see that this term also tends to 0 as $t \rightarrow \infty$. Thus we have (2.2). The proof of Lemma 2.1 is complete.

Theorem 2.1. Consider equation (2.1) and suppose that (1.3), or equivalently $(2.2)$, holds. Then $(2.1)$ has a solution $x(t)$ on $[a, \infty)$ such that

$$
x(t) \neq 0 \text { for } t \in[T, \infty) \text {, where } T \geq a,
$$

and

$$
\lim _{t \rightarrow \infty} \frac{x(t)}{\sqrt{t}}=0 \quad \text { and } \quad \lim _{t \rightarrow \infty} \sqrt{t} x^{\prime}(t)=0 .
$$

Proof. We utilize (2.2), instead of (1.3), for the proof. Since (2.2) holds, it is possible to choose a $T \geq a$ such that

$$
t^{\alpha / 2} \int_{t}^{\infty} s^{\alpha / 2} p(s) d s \leq 2^{-\alpha}, \quad t \geq T .
$$

For this $T$, we take a positive number $k$ such that $k \sqrt{T} \geq 1$, and fix these numbers $T$ and $k$.

Let $C[T, \infty)$ be the Fréchet space that consists of all continuous functions on $[T, \infty)$ with the topology of uniform convergence on compact subintervals of $[T, \infty)$. Further, let $X$ be the following subset of $C[T, \infty)$ :

$$
X=\{x \in C[T, \infty): 1 \leq x(t) \leq k \sqrt{t}, \quad t \geq T\} .
$$

Then we consider the mapping $M: X \rightarrow C[T, \infty)$ defined by

$$
(M x)(t)=1+\int_{T}^{t}\left(\int_{s}^{\infty} p(r)(x(r))^{\alpha} d r\right)^{1 / \alpha} d s, \quad t \geq T .
$$

By the first condition of (2.2), $M$ is well defined on $X$. We will show by the Schauder-Tychonoff theorem that $M$ has a fixed point $x=x(t)$ in $X$.

Let $x \in X$. Clearly, $(M x)(t)$ is a continuous function of $t \in[T, \infty)$. From (2.7) it follows that

$$
0 \leq \int_{s}^{\infty} p(r)(x(r))^{\alpha} d r \leq k^{\alpha} \int_{s}^{\infty} r^{\alpha / 2} p(r) d r \leq 2^{-\alpha} k^{\alpha} s^{-\alpha / 2}, \quad s \geq T .
$$


Further, we have

$$
\begin{aligned}
0 \leq \int_{T}^{t}\left(\int_{s}^{\infty} p(r)(x(r))^{\alpha} d r\right)^{1 / \alpha} d s & \leq 2^{-1} k \int_{T}^{t} s^{-1 / 2} d s \\
& =k \sqrt{t}-k \sqrt{T} \leq k \sqrt{t}-1, \quad t \geq T,
\end{aligned}
$$

and hence we see that $1 \leq(M x)(t) \leq k \sqrt{t}$ for $t \geq T$. Thus $x \in X$ implies $M x \in X$, that is, $M$ maps $X$ into $X$.

By the Lebesgue dominated convergence theorem, it is easily verified that if $x, x_{j} \in X(j=1,2, \cdots)$ and $\lim _{j \rightarrow \infty} x_{j}(t)=x(t)$ uniformly on every compact subinterval of $[T, \infty)$, then also $\lim _{j \rightarrow \infty}\left(M x_{j}\right)(t)=(M x)(t)$ uniformly on every compact subinterval of $[T, \infty)$. Thus $M$ is a continuous operator on $X$.

Since $M(X) \subset X$, we see that $\{(M x)(t): x \in X\}$ is uniformly bounded on each compact subinterval of $[T, \infty)$. Noting that

$$
(M x)^{\prime}(t)=\left(\int_{t}^{\infty} p(r)(x(r))^{\alpha} d r\right)^{1 / \alpha}, \quad t \geq T,
$$

and using the estimate (2.8), we find that $\left\{(M x)^{\prime}(t): x \in X\right\}$ is also uniformly bounded on each compact subinterval of $[T, \infty)$. Then the Ascoli-Arzelà theorem implies that $M(X)$ has compact closure in the topology of $C[T, \infty)$.

Thus all the hypotheses of the Schauder-Tychonoff fixed point theorem have been verified to hold, and so there exists an element $x=x(t) \in X$ such that $x=M x$, that is,

$$
x(t)=1+\int_{T}^{t}\left(\int_{s}^{\infty} p(r)(x(r))^{\alpha} d r\right)^{1 / \alpha} d s, \quad t \geq T .
$$

Differentiation of (2.9) shows that $x(t)$ is a solution of $(2.1)$ on the interval $[T, \infty)$. Continuing $x(t)$ to the left of $T$ as a solution of (2.1) (which is possible by a continuation theorem [16, Lemma 2.1]), we obtain a solution of (2.1) defined on all of the interval $[a, \infty)$. This solution existing on $[a, \infty)$ is denoted by $x(t)$ again.

It is trivial that $x(t) \geq 1$ for $t \geq T$. Since $x(t) \leq k \sqrt{t}$ for $t \geq T$, it follows from the second condition in (2.2) that

$$
\begin{aligned}
0 \leq \sqrt{t} x^{\prime}(t) & =\sqrt{t}\left(\int_{t}^{\infty} p(r)(x(r))^{\alpha} d r\right)^{1 / \alpha} \\
& \leq k\left(t^{\alpha / 2} \int_{t}^{\infty} r^{\alpha / 2} p(r) d r\right)^{1 / \alpha} \\
& \rightarrow 0 \text { as } t \rightarrow \infty,
\end{aligned}
$$

and consequently $\lim _{t \rightarrow \infty} \sqrt{t} x^{\prime}(t)=0$. Then, by l'Hospital's rule we see that

$$
\lim _{t \rightarrow \infty} \frac{x(t)}{\sqrt{t}}=\lim _{t \rightarrow \infty} \frac{x^{\prime}(t)}{(1 / 2) t^{-1 / 2}}=0 .
$$

Thus $x(t)$ is a solution of $(2.1)$ on the interval $[a, \infty)$ satisfying the properties $(2.5)$ and (2.6). The proof of Theorem 2.1 is complete.

Remark 2.1. By the proof of Theorem 2.1 we see that if (1.3) or (2.2) holds and if $T \geq a$ satisfies (2.7), then equation (2.1) has a solution $x(t)$ that does not vanish 
on $[T, \infty)$ and satisfies $1 \leq x(t) \leq k \sqrt{t}$ for $t \geq T$, where $k$ is chosen so large that $k \sqrt{T} \geq 1$.

The following theorem is concerned with the essential uniqueness of a nonoscillatory solution $x(t)$ of (2.1) having the property

$$
\int^{\infty} \frac{d t}{(x(t))^{2}}=+\infty
$$

Theorem 2.2. Let $\alpha \geq 1$. Suppose that (2.1) is nonoscillatory. Then, a nonoscillatory solution $x(t)$ of (2.1) satisfying (2.10) is uniquely determined up to a nonzero constant factor.

Proof. Let $x=x_{1}(t)$ and $x=x_{2}(t)$ be eventually positive solutions of (2.1) satisfying

$$
\int^{\infty} \frac{d t}{\left(x_{1}(t)\right)^{2}}=+\infty \text { and } \int^{\infty} \frac{d t}{\left(x_{2}(t)\right)^{2}}=+\infty
$$

respectively. Take $T(\geq a)$ so large that $x_{i}(t)>0$ for $t \in[T, \infty), i=1,2$, and put

$$
y_{i}(t)=\frac{x_{i}(t)}{x_{i}(T)}, \quad t \in[T, \infty), \quad i=1,2 .
$$

Then $y_{i}(t), i=1,2$, are solutions of (2.1) satisfying $y_{i}(T)=1, y_{i}(t)>0$ for $t \geq T$ $(i=1,2)$, and

$$
\int_{T}^{\infty} \frac{d t}{\left(y_{1}(t)\right)^{2}}=+\infty \text { and } \int_{T}^{\infty} \frac{d t}{\left(y_{2}(t)\right)^{2}}=+\infty .
$$

By the equation (2.1) it is seen that $y_{i}^{\prime}(t)>0$ for $t \geq T$, so that $y_{i}(t), i=1,2$, satisfy

$$
\left(\left(y_{i}^{\prime}(t)\right)^{\alpha}\right)^{\prime}+p(t)\left(y_{i}(t)\right)^{\alpha}=0, \quad t \geq T .
$$

Therefore, $y_{i}(t)(i=1,2)$ are in class $C^{2}$ and

$$
y_{i}^{\prime \prime}(t)=-\frac{1}{\alpha} p(t)\left(y_{i}(t)\right)^{\alpha}\left(y_{i}^{\prime}(t)\right)^{1-\alpha}, \quad t \geq T .
$$

In the following we claim that $y_{1}^{\prime}(T)=y_{2}^{\prime}(T)$. To prove this by contradiction, assume the contrary. Then there is no loss of generality in assuming that $y_{1}^{\prime}(T)<$ $y_{2}^{\prime}(T)$. Let us denote by $W(t)$ the Wronskian of $y_{1}(t)$ and $y_{2}(t)$ :

$$
W(t)=y_{1}(t) y_{2}^{\prime}(t)-y_{1}^{\prime}(t) y_{2}(t), \quad t \geq T .
$$

We have $W(T)=y_{2}^{\prime}(T)-y_{1}^{\prime}(T)>0$, and it follows from (2.11) that

$$
W^{\prime}(t)=-\frac{1}{\alpha} p(t) y_{1}(t) y_{2}(t)\left\{\left(\frac{y_{2}(t)}{y_{2}^{\prime}(t)}\right)^{\alpha-1}-\left(\frac{y_{1}(t)}{y_{1}^{\prime}(t)}\right)^{\alpha-1}\right\}, \quad t \geq T .
$$

In the case $\alpha=1$, we have $W^{\prime}(t) \equiv 0(t \geq T)$; hence $W(t)=W(T)(t \geq T)$. In the case $\alpha>1$, we see that

$$
W^{\prime}(T)=-\frac{1}{\alpha} p(T)\left\{\left(y_{2}^{\prime}(T)\right)^{1-\alpha}-\left(y_{1}^{\prime}(T)\right)^{1-\alpha}\right\}>0 .
$$

If $W^{\prime}(t) \leq 0$ for some $t \in(T, \infty)$, then there is a number $S \in(T, \infty)$ such that $W^{\prime}(S)=0$ and $W^{\prime}(t)>0$ for $t \in[T, S)$. Then it follows from (2.12) that

$$
\left(\frac{y_{2}(S)}{y_{2}^{\prime}(S)}\right)^{\alpha-1}=\left(\frac{y_{1}(S)}{y_{1}^{\prime}(S)}\right)^{\alpha-1}
$$


which yields $W(S)=0$. This is a contradiction, since $W(T)>0$ and $W^{\prime}(t)>0$ for $t \in[T, S)$. Consequently we find that $W^{\prime}(t)>0$ for all $t \in(T, \infty)$. Therefore, we have

$$
W(t) \geq W(T)>0, \quad t \geq T
$$

in both the cases $\alpha=1$ and $\alpha>1$. Then

$$
\left(\frac{y_{1}(t)}{y_{2}(t)}\right)^{\prime}=-\frac{W(t)}{\left(y_{2}(t)\right)^{2}} \leq-\frac{W(T)}{\left(y_{2}(t)\right)^{2}}, \quad t \geq T .
$$

Integrating the above inequality on $[T, t]$ and letting $t \rightarrow \infty$, we see that

$$
\frac{y_{1}(t)}{y_{2}(t)}-1 \leq-W(T) \int_{T}^{t} \frac{d s}{\left(y_{2}(s)\right)^{2}} \rightarrow-\infty \quad \text { as } t \rightarrow \infty .
$$

This is a contradiction, because $y_{1}(t)$ and $y_{2}(t)$ are positive on $[T, \infty)$. Thus it has been proved that $y_{1}^{\prime}(T)=y_{2}^{\prime}(T)$.

Since $y_{1}(t)$ and $y_{2}(t)$ take the same initial values $y_{1}(T)=y_{2}(T)=1$ and $y_{1}^{\prime}(T)=$ $y_{2}^{\prime}(T)$, these solutions are identical: $y_{1}(t) \equiv y_{2}(t)$ for $t \in[a, \infty)$. Thus we have $x_{1}(t)=c x_{2}(t)$ for $t \in[a, \infty)$ with $c=x_{1}(T) / x_{2}(T)>0$. The proof of Theorem 2.2 is complete.

Remark 2.2. For the linear equation (1.2), Theorem 2.2 is well known. Moreover, it is also known that if (1.2) is nonoscillatory, then (1.2) always has a nonoscillatory solution $x(t)$ satisfying (2.10). Such a solution $x(t)$ is called a principal solution of (1.2) $($ at $t=\infty)$. For an excellent account of principal solutions to linear equations, see Hartman [7, p. 355].

The following theorem gives a comparison result for the two equations

$$
\left(\left|x^{\prime}\right|^{\alpha} \operatorname{sgn} x^{\prime}\right)^{\prime}+p(t)|x|^{\alpha} \operatorname{sgn} x=0, \quad t \geq a,
$$

and

$$
\left(\left|y^{\prime}\right|^{\alpha} \operatorname{sgn} y^{\prime}\right)^{\prime}+P(t)|y|^{\alpha} \operatorname{sgn} y=0, \quad t \geq a .
$$

Theorem 2.3. Let $\alpha \geq 1$. Suppose that $p(t)$ and $P(t)$ are continuous functions on $[a, \infty), a>0$, such that $0<p(t) \leq P(t), p(t) \equiv P(t)$, for $t \in[a, \infty)$. Let $x(t)$ and $y(t)$ be eventually positive solutions of (2.13) and (2.14) satisfying

$$
\begin{gathered}
x(t)>0(t \in[T, \infty)), \quad \int^{\infty} \frac{d t}{(x(t))^{2}}=+\infty, \quad \text { and } \\
y(t)>0(t \in[T, \infty)), \quad \int^{\infty} \frac{d t}{(y(t))^{2}}=+\infty,
\end{gathered}
$$

respectively. Then we have

$$
\begin{gathered}
0<\frac{x(t)}{x(T)}<\frac{y(t)}{y(T)}, \quad t>T, \quad \text { and } \\
0<\frac{x^{\prime}(t)}{x(t)}<\frac{y^{\prime}(t)}{y(t)}, \quad t \geq T .
\end{gathered}
$$


Proof. We put

$$
y_{0}(t)=\frac{y(t)}{y(T)}, \quad t \geq T .
$$

Then, as in the proof of Theorem 2.2, we see that $y_{0}(t)$ is a solution of (2.14) having the properties that $y_{0}(T)=1$ and $y_{0}(t)>0, y_{0}^{\prime}(t)>0$ and $y_{0}^{\prime \prime}(t)<0$ for $t \geq T$, and

$$
\int^{\infty} \frac{d t}{\left(y_{0}(t)\right)^{2}}=+\infty
$$

If $\lim _{t \rightarrow \infty} y_{0}^{\prime}(t)$ has a positive finite limit, then (2.19) is not satisfied. Therefore, we have $\lim _{t \rightarrow \infty} y_{0}^{\prime}(t)=0$, and so

$$
y_{0}^{\prime}(t)=\left(\int_{t}^{\infty} P(s)\left(y_{0}(s)\right)^{\alpha} d s\right)^{1 / \alpha}, \quad t \geq T .
$$

An integration of this equality gives

$$
y_{0}(t)=1+\int_{T}^{t}\left(\int_{s}^{\infty} P(r)\left(y_{0}(r)\right)^{\alpha} d r\right)^{1 / \alpha} d s, \quad t \geq T .
$$
by

Consider the subset $X$ of $C[T, \infty)$ and the mapping $M: X \rightarrow C[T, \infty)$ defined

$$
X=\left\{x \in C[T, \infty): 1 \leq x(t) \leq y_{0}(t), \quad t \geq T\right\}
$$

and

$$
(M x)(t)=1+\int_{T}^{t}\left(\int_{s}^{\infty} p(r)(x(r))^{\alpha} d r\right)^{1 / \alpha} d s, \quad t \geq T,
$$

respectively. As in the proof of Theorem 2.1, we can show that all the hypotheses of the Schauder-Tychonoff fixed point theorem are satisfied. Let $x=x_{0} \in X$ be a fixed point of $M$. We have

$$
x_{0}(t)=1+\int_{T}^{t}\left(\int_{s}^{\infty} p(r)\left(x_{0}(r)\right)^{\alpha} d r\right)^{1 / \alpha} d s, \quad t \geq T,
$$

and so $x_{0}(t)$ is a positive solution of $(2.13)$ on $[T, \infty)$ such that $x_{0}(T)=1$. We continue $x_{0}(t)$ to the left of $T$ as a solution of (2.13), and denote the solution existing on all of $[a, \infty)$ by $x_{0}(t)$ again. Since $x_{0}(t)$ satisfies

$$
1 \leq x_{0}(t) \leq y_{0}(t), \quad t \geq T,
$$

the conditions $p(t) \leq P(t)$ and $p(t) \not \equiv P(t)$ yield

$$
0<x_{0}^{\prime}(t)=\left(\int_{t}^{\infty} p(s)\left(x_{0}(s)\right)^{\alpha} d s\right)^{1 / \alpha}<\left(\int_{t}^{\infty} P(s)\left(y_{0}(s)\right)^{\alpha} d s\right)^{1 / \alpha}=y_{0}^{\prime}(t)
$$

for $t \geq T$, from which it follows that

$$
1 \leq x_{0}(t)<y_{0}(t), \quad t>T .
$$

By (2.19) and (2.21) we have

$$
\int^{\infty} \frac{d t}{\left(x_{0}(t)\right)^{2}}=+\infty
$$


and hence Theorem 2.2 shows that $x_{0}(t)$ is a positive constant multiple of $x(t)$. Thus

$$
x_{0}(t)=\frac{x(t)}{x(T)}, \quad t \geq T .
$$

Then (2.21) gives (2.17). Further, (2.20) yields

$$
0<\frac{x^{\prime}(t)}{x(T)}<\frac{y^{\prime}(t)}{y(T)}, \quad t \geq T
$$

and, in particular, we have

$$
0<\frac{x^{\prime}(T)}{x(T)}<\frac{y^{\prime}(T)}{y(T)} .
$$

Then, since $T$ can be an arbitrary number such that $x(t)>0$ and $y(t)>0$ for $t \geq T$, we conclude that (2.18) is satisfied. The proof of Theorem 2.3 is complete.

\section{Proofs of the Main Results}

In this section we present the proofs of Theorems 1.1 and 1.2.

Proof of Theorem 1.1. Let $\alpha>0$. Then Theorem 2.1 applied to the equation $\left(\mathrm{H}_{\lambda}\right)$ shows that, for each $\lambda>0$, there is a solution $x=x_{\lambda}(t)$ of $\left(\mathrm{H}_{\lambda}\right)$ such that

$$
x_{\lambda}(t) \neq 0 \text { for } t \in[T, \infty) \text {, where } T \geq a,
$$

and

$$
\lim _{t \rightarrow \infty} \frac{x_{\lambda}(t)}{\sqrt{t}}=0 \quad \text { and } \quad \lim _{t \rightarrow \infty} \sqrt{t} x_{\lambda}^{\prime}(t)=0 .
$$

Here $T=T_{\lambda}$ depends on $\lambda>0$. Observe that this solution $x=x_{\lambda}(t)$ of $\left(\mathrm{H}_{\lambda}\right)$ satisfies (2.10), and so we conclude by Theorem 2.2 that if $\alpha \geq 1$, then $x_{\lambda}(t)$ is uniquely determined up to a nonzero constant multiple. This proves Theorem 1.1 .

In what follows, we restrict our attention to the case $\alpha \geq 1$ and assume that $p(t)$ is a continuous function on $[a, \infty), a>0$, and $p(t)>0$ for $t \in[a, \infty)$, and moreover that $p(t)$ satisfies (1.3), or equivalently (2.2). To fix the idea, we normalize the solutions $x_{\lambda}(t)$ of $\left(\mathrm{H}_{\lambda}\right)$ which are obtained in Theorem 1.1 by the following conditions:

$$
\left(x_{\lambda}(a)\right)^{2}+\left(x_{\lambda}^{\prime}(a)\right)^{2}=1 \quad \text { and } \quad x_{\lambda}(t)>0 \text { for all large } t .
$$

We denote this normalized solution by $x(t ; \lambda)$. Thus $x(t ; \lambda)$ gives a precisely unique solution of $\left(\mathrm{H}_{\lambda}\right)$ such that

$$
\begin{gathered}
x(t ; \lambda)>0 \text { for } t \geq T, \text { where } T(\geq a) \text { depends on } \lambda>0, \\
(x(a ; \lambda))^{2}+\left(x^{\prime}(a ; \lambda)\right)^{2}=1, \quad \text { and } \\
\lim _{t \rightarrow \infty} \frac{x(t ; \lambda)}{\sqrt{t}}=0 .
\end{gathered}
$$

Further, by (3.2), we have

$$
\lim _{t \rightarrow \infty} \sqrt{t} x^{\prime}(t ; \lambda)=0
$$


It is also verified that

$$
\begin{gathered}
\lim _{\lambda \rightarrow+0} x(a ; \lambda)=1 \quad \text { and } \\
\lim _{\lambda \rightarrow+0} x^{\prime}(a ; \lambda)=0 .
\end{gathered}
$$

In fact, we can take a number $\lambda_{*}>0$ such that

$$
\lambda_{*} t^{\alpha / 2} \int_{t}^{\infty} s^{\alpha / 2} p(s) d s \leq 2^{-\alpha}, \quad t \geq a .
$$

Then, by Remark 2.1 applied to $\left(\mathrm{H}_{\lambda}\right)$, we see that

$$
\text { if } \lambda \in\left(0, \lambda_{*}\right] \text {, then } 0<x(t ; \lambda) \leq k \sqrt{t} \text { for } t \in[a, \infty) \text {, }
$$

where $k>0$ is chosen so large that $k \sqrt{a} \geq 1$. Therefore,

$$
\begin{aligned}
0<x^{\prime}(a ; \lambda) & =\left(\lambda \int_{a}^{\infty} p(s)(x(s ; \lambda))^{\alpha} d s\right)^{1 / \alpha} \\
& \leq k \lambda^{1 / \alpha}\left(\int_{a}^{\infty} s^{\alpha / 2} p(s) d s\right)^{1 / \alpha} \\
& \rightarrow 0 \quad \text { as } \lambda \rightarrow+0
\end{aligned}
$$

and, by the normalized condition (3.4),

$$
x(a ; \lambda)=\left(1-\left(x^{\prime}(a ; \lambda)\right)^{2}\right)^{1 / 2} \rightarrow 1 \text { as } \lambda \rightarrow+0 .
$$

As an immediate consequence of (2.17) and (2.18) in Theorem 2.3 we see that, if $0<\lambda<\mu$ and if $x(t ; \lambda)>0$ and $x(t ; \mu)>0$ for $t \geq T$, then

$$
0<\frac{x(t ; \lambda)}{x(T ; \lambda)}<\frac{x(t ; \mu)}{x(T ; \mu)}, \quad t>T,
$$

and

$$
0<\frac{x^{\prime}(t ; \lambda)}{x(t ; \lambda)}<\frac{x^{\prime}(t ; \mu)}{x(t ; \mu)}, \quad t \geq T .
$$

Theorem 3.1. For each fixed $t \in[a, \infty), x(t ; \lambda)$ and $x^{\prime}(t ; \lambda)$ are continuous functions of $\lambda \in(0, \infty)$.

Proof. Let $\lambda=\lambda_{0} \in(0, \infty)$ be fixed. We will show that $x(t ; \lambda)$ and $x^{\prime}(t ; \lambda)$ are continuous at $\lambda=\lambda_{0}$ for each $t \in[a, \infty)$. Choose $T \geq a$ so large that

$$
\left(\lambda_{0}+1\right) t^{\alpha / 2} \int_{t}^{\infty} s^{\alpha / 2} p(s) d s \leq 2^{-\alpha}, \quad t \geq T .
$$

Then, by Remark 2.1 applied to $\left(\mathrm{H}_{\lambda}\right)$, we find that, for any $\lambda \in\left(0, \lambda_{0}+1\right], x(t ; \lambda)$ is positive on $[T, \infty)$. Note here that the number $T$ does not depend on $\lambda \in\left(0, \lambda_{0}+1\right]$. For $\lambda \in\left(0, \lambda_{0}+1\right]$, we define the function $y(t ; \lambda)$ by

$$
y(t ; \lambda)=\frac{x(t ; \lambda)}{x(T ; \lambda)}, \quad t \geq a .
$$

Then $y(t ; \lambda)$ is a solution of $\left(\mathrm{H}_{\lambda}\right)$, where $0<\lambda \leq \lambda_{0}+1$, such that $y(t ; \lambda)>0$ for $t \geq T$ and $y(T ; \lambda)=1$ and $\lim _{t \rightarrow \infty} y(t ; \lambda) / \sqrt{t}=0$. Let

$$
\gamma(\lambda)=y^{\prime}(T ; \lambda)=\frac{x^{\prime}(T ; \lambda)}{x(T ; \lambda)} .
$$


By (3.10) and (3.11), $y(t ; \lambda)$ is strictly increasing in $\lambda \in\left(0, \lambda_{0}+1\right]$ for each fixed $t \in(T, \infty)$, and $\gamma(\lambda)$ is also strictly increasing in $\lambda \in\left(0, \lambda_{0}+1\right]$.

Now let us verify that if $\left\{\lambda_{n}\right\}_{n=1}^{\infty}$ is a sequence such that

$$
\lambda_{1}>\lambda_{2}>\cdots>\lambda_{n}>\cdots>\lambda_{0} \text { and } \lim _{n \rightarrow \infty} \lambda_{n}=\lambda_{0},
$$

then $\lim _{n \rightarrow \infty} \gamma\left(\lambda_{n}\right)=\gamma\left(\lambda_{0}\right)$. In fact, the strict increasing property of $y(t ; \lambda)$ and $\gamma(\lambda)$ in $\lambda \in\left(0, \lambda_{0}+1\right]$ implies

$$
\begin{gathered}
y\left(t ; \lambda_{1}\right)>y\left(t ; \lambda_{2}\right)>\cdots>y\left(t ; \lambda_{n}\right)>\cdots>y\left(t ; \lambda_{0}\right) \text { for } t>T, \text { and } \\
\gamma\left(\lambda_{1}\right)>\gamma\left(\lambda_{2}\right)>\cdots>\gamma\left(\lambda_{n}\right)>\cdots>\gamma\left(\lambda_{0}\right) .
\end{gathered}
$$

By (3.13), $\left\{\gamma\left(\lambda_{n}\right)\right\}_{n=1}^{\infty}$ has a positive finite limit as $n \rightarrow \infty$. We denote this limit by $\gamma_{0}$ :

$$
\gamma_{0}=\lim _{n \rightarrow \infty} \gamma\left(\lambda_{n}\right)
$$

For each $n=1,2, \cdots, y\left(t ; \lambda_{n}\right)$ is a solution of the initial value problem

$$
\left\{\begin{array}{l}
\left(\left|u^{\prime}\right|^{\alpha} \operatorname{sgn} u^{\prime}\right)^{\prime}+\lambda_{n} p(t)|u|^{\alpha} \operatorname{sgn} u=0, \quad t \geq a, \\
u(T)=1 \text { and } u^{\prime}(T)=\gamma\left(\lambda_{n}\right) .
\end{array}\right.
$$

Let $y_{0}(t)$ be a solution of the initial value problem

$$
\left\{\begin{array}{l}
\left(\left|u^{\prime}\right|^{\alpha} \operatorname{sgn} u^{\prime}\right)^{\prime}+\lambda_{0} p(t)|u|^{\alpha} \operatorname{sgn} u=0, \quad t \geq a, \\
u(T)=1 \text { and } u^{\prime}(T)=\gamma_{0} .
\end{array}\right.
$$

Since $\lambda_{n} \rightarrow \lambda_{0}(n \rightarrow \infty)$ and $\gamma\left(\lambda_{n}\right) \rightarrow \gamma_{0}(n \rightarrow \infty)$, it follows from a general fundamental theorem (Coppel [2, Theorem 3, p. 17] or Coddington and Levinson $\left[1\right.$, Theorem 4.3, p. 59]) that $\left\{y\left(t ; \lambda_{n}\right)\right\}$ converges to $y_{0}(t)$ uniformly on each compact subinterval of $[a, \infty)$. By $(3.12)$ we have $y\left(t ; \lambda_{1}\right)>y_{0}(t) \geq y\left(t ; \lambda_{0}\right)$ for $t>T$, and so $y_{0}(t)$ satisfies

$$
\lim _{t \rightarrow \infty} \frac{y_{0}(t)}{\sqrt{t}}=0 .
$$

Since both $x=y_{0}(t)$ and $x=y\left(t ; \lambda_{0}\right)$ are solutions of $\left(\mathrm{H}_{\lambda}\right)$ with $\lambda=\lambda_{0}$ and satisfy (2.10), we can conclude by Theorem 2.2 that $y_{0}(t)$ is a positive constant multiple of $y\left(t ; \lambda_{0}\right)$. Then, in view of $y_{0}(T)=y\left(T ; \lambda_{0}\right)=1$, we get $y_{0}(t) \equiv y\left(t ; \lambda_{0}\right)$ for $t \in[a, \infty)$. Thus,

$$
\lim _{n \rightarrow \infty} \gamma\left(\lambda_{n}\right)=\gamma_{0}=y_{0}^{\prime}(T)=y^{\prime}\left(T ; \lambda_{0}\right)=\gamma\left(\lambda_{0}\right) .
$$

As in the above discussion, it is verified that if $\left\{\lambda_{n}\right\}_{n=1}^{\infty}$ is a sequence such that

$$
0<\lambda_{1}<\lambda_{2}<\cdots<\lambda_{n}<\cdots<\lambda_{0} \text { and } \lim _{n \rightarrow \infty} \lambda_{n}=\lambda_{0},
$$

then $\lim _{n \rightarrow \infty} \gamma\left(\lambda_{n}\right)=\gamma\left(\lambda_{0}\right)$. Consequently, we conclude that, for any sequence $\left\{\lambda_{n}\right\}_{n=1}^{\infty}$ with $\lim _{n \rightarrow \infty} \lambda_{n}=\lambda_{0}$, the following is true:

$$
\lim _{n \rightarrow \infty} \gamma\left(\lambda_{n}\right)=\gamma\left(\lambda_{0}\right)
$$


Now, let $\left\{\lambda_{n}\right\}_{n=1}^{\infty}$ be any sequence satisfying $\lim _{n \rightarrow \infty} \lambda_{n}=\lambda_{0}$. We consider the initial value problem $\left(3.14_{n}\right), n=1,2, \cdots$, and

$$
\left\{\begin{array}{l}
\left(\left|u^{\prime}\right|^{\alpha} \operatorname{sgn} u^{\prime}\right)^{\prime}+\lambda_{0} p(t)|u|^{\alpha} \operatorname{sgn} u=0, \quad t \geq a \\
u(T)=1 \text { and } u^{\prime}(T)=\gamma\left(\lambda_{0}\right) .
\end{array}\right.
$$

Note that $y\left(t ; \lambda_{n}\right)$ is a solution of $\left(3.14_{n}\right), n=1,2, \cdots$, and $y\left(t ; \lambda_{0}\right)$ is a solution of (3.16). Since $\lambda_{n} \rightarrow \lambda_{0}(n \rightarrow \infty)$ and $\gamma\left(\lambda_{n}\right) \rightarrow \gamma\left(\lambda_{0}\right)(n \rightarrow \infty)$, from the general fundamental theorem, which has been used in the previous argument, it follows that $y\left(t ; \lambda_{n}\right) \rightarrow y\left(t ; \lambda_{0}\right)$ and $y^{\prime}\left(t ; \lambda_{n}\right) \rightarrow y^{\prime}\left(t ; \lambda_{0}\right)$ as $n \rightarrow \infty$ uniformly on each compact subinterval of $[a, \infty)$. This means that

$$
\frac{x\left(t ; \lambda_{n}\right)}{x\left(T ; \lambda_{n}\right)} \rightarrow \frac{x\left(t ; \lambda_{0}\right)}{x\left(T ; \lambda_{0}\right)} \quad(n \rightarrow \infty)
$$

and

$$
\frac{x^{\prime}\left(t ; \lambda_{n}\right)}{x\left(T ; \lambda_{n}\right)} \rightarrow \frac{x^{\prime}\left(t ; \lambda_{0}\right)}{x\left(T ; \lambda_{0}\right)} \quad(n \rightarrow \infty)
$$

uniformly on each compact subinterval of $[a, \infty)$. Then (3.17) and (3.18), together with the normalizing condition

$$
\left(x\left(a ; \lambda_{n}\right)\right)^{2}+\left(x^{\prime}\left(a ; \lambda_{n}\right)\right)^{2}=\left(x\left(a ; \lambda_{0}\right)\right)^{2}+\left(x^{\prime}\left(a ; \lambda_{0}\right)\right)^{2}=1,
$$

imply

$$
\begin{aligned}
\frac{1}{\left(x\left(T ; \lambda_{n}\right)\right)^{2}} & =\left(\frac{x\left(a ; \lambda_{n}\right)}{x\left(T ; \lambda_{n}\right)}\right)^{2}+\left(\frac{x^{\prime}\left(a ; \lambda_{n}\right)}{x\left(T ; \lambda_{n}\right)}\right)^{2} \\
& \rightarrow\left(\frac{x\left(a ; \lambda_{0}\right)}{x\left(T ; \lambda_{0}\right)}\right)^{2}+\left(\frac{x^{\prime}\left(a ; \lambda_{0}\right)}{x\left(T ; \lambda_{0}\right)}\right)^{2}=\frac{1}{\left(x\left(T ; \lambda_{0}\right)\right)^{2}}
\end{aligned}
$$

as $n \rightarrow \infty$, and hence

$$
x\left(T ; \lambda_{n}\right) \rightarrow x\left(T ; \lambda_{0}\right) \quad \text { as } \quad n \rightarrow \infty .
$$

Then, by (3.17) and (3.18), it is easy to see that

$$
x\left(t ; \lambda_{n}\right) \rightarrow x\left(t ; \lambda_{0}\right) \quad \text { and } \quad x^{\prime}\left(t ; \lambda_{n}\right) \rightarrow x^{\prime}\left(t ; \lambda_{0}\right) \quad \text { as } n \rightarrow \infty
$$

uniformly on each compact subinterval of $[a, \infty)$. In particular, $x(t ; \lambda)$ and $x^{\prime}(t ; \lambda)$ are continuous at $\lambda=\lambda_{0} \in(0, \infty)$ for each fixed $t \in[a, \infty)$. The proof of Theorem 3.1 is complete.

Now let us define the generalized trigonometric functions $S(\tau), C(\tau)$ and $T(\tau)$ which generalize the classical trigonometric functions $\sin \tau, \cos \tau$ and $\tan \tau$, respectively. The generalized trigonometric functions are used to extend in a natural way the notion of the Prüfer transformation, known for the Sturm-Liouville equation, to the half-linear equation $\left(\mathrm{H}_{\lambda}\right)$. These generalized functions were introduced by Elbert [4]. For the properties stated below, see [4].

The generalized sine function $S=S(\tau)$ is defined as the solution of the specific half-linear equation

$$
\left(|\dot{S}|^{\alpha} \operatorname{sgn} \dot{S}\right)^{\cdot}+\alpha|S|^{\alpha} \operatorname{sgn} S=0 \quad\left(\cdot=\frac{d}{d \tau}\right)
$$

satisfying the initial conditions

$$
S(0)=0, \dot{S}(0)=1
$$


The generalized sine function $S(\tau)$ has the same properties as the classical sine function $\sin \tau$. First of all, it is defined on $\boldsymbol{R}$ and is periodic with period $2 \pi_{\alpha}$, where

$$
\pi_{\alpha}=\frac{2 \pi}{\alpha+1} / \sin \frac{\pi}{\alpha+1}
$$

Further, $S(\tau)$ is an odd function having zeros at $\tau=j \pi_{\alpha}, j \in \boldsymbol{Z}$; it is positive on the intervals $2 j \pi_{\alpha}<\tau<(2 j+1) \pi_{\alpha}, j \in \boldsymbol{Z}$, and negative on the intervals $(2 j+1) \pi_{\alpha}<\tau<2(j+1) \pi_{\alpha}, j \in Z$. Thus,

$$
S(\tau)=0 \quad \text { if and only if } \tau=j \pi_{\alpha} \text { for some } j \in \boldsymbol{Z} \text {. }
$$

The generalized cosine function $C(\tau)$ is the derivative $\dot{S}(\tau)$ of $S(\tau): C(\tau)=\dot{S}(\tau)$. The $C(\tau)$ is periodic with period $2 \pi_{\alpha}$, and is an even function. It has zeros at $\tau=$ $\left(j+\frac{1}{2}\right) \pi_{\alpha}, j \in Z$, and is positive for $\left(2 j-\frac{1}{2}\right) \pi_{\alpha}<\tau<\left(2 j+\frac{1}{2}\right) \pi_{\alpha}, j \in Z$, and negative for $\left(2 j+\frac{1}{2}\right) \pi_{\alpha}<\tau<\left(2 j+\frac{3}{2}\right) \pi_{\alpha}, j \in \boldsymbol{Z}$. We have

$$
C(\tau)=0 \quad \text { if and only if } \tau=\left(j+\frac{1}{2}\right) \pi_{\alpha} \text { for some } j \in Z \text {. }
$$

Moreover, the generalized Pythagorean theorem holds for $S(\tau)$ and $C(\tau)$ :

$$
|S(\tau)|^{\alpha+1}+|C(\tau)|^{\alpha+1}=1 \text { for all } \tau .
$$

The generalized tangent function $T(\tau)$ is defined by

$$
T(\tau)=\frac{S(\tau)}{C(\tau)} \quad \text { for } \tau \neq\left(j+\frac{1}{2}\right) \pi_{\alpha}, j \in \boldsymbol{Z} .
$$

It is periodic with period $\pi_{\alpha}$ and satisfies

$$
\dot{T}=1+|T|^{\alpha+1}>0 \quad \text { for } \tau \neq\left(j+\frac{1}{2}\right) \pi_{\alpha}, j \in Z,
$$

so that $T(\tau)$ is strictly increasing for $\left(j-\frac{1}{2}\right) \pi_{\alpha}<\tau<\left(j+\frac{1}{2}\right) \pi_{\alpha}, j \in \boldsymbol{Z}$. We have

$$
\begin{aligned}
& \lim T(\tau)=-\infty \quad \text { as } \tau \rightarrow\left(j-\frac{1}{2}\right) \pi_{\alpha}+0, \text { and } \\
& \lim T(\tau)=+\infty \quad \text { as } \tau \rightarrow\left(j+\frac{1}{2}\right) \pi_{\alpha}-0 .
\end{aligned}
$$

Lemma 3.1. (i) There is $\lambda_{*}>0$ such that if $0<\lambda<\lambda_{*}$, then $x(t ; \lambda)>0$ for $t \in[a, \infty)$.

(ii) For any $N \in \boldsymbol{N}$, there is $\lambda^{*}>0$ such that if $\lambda>\lambda^{*}$, then $x(t ; \lambda)$ has at least $N$ zeros in the interval $[a, a+1]$.

Proof. The first part (i) is an immediate consequence of (3.9). To prove the second part (ii), we consider the constant coefficient equation

$$
\left(\left|y^{\prime}\right|^{\alpha} \operatorname{sgn} y^{\prime}\right)^{\prime}+\alpha \mu^{\alpha+1}|y|^{\alpha} \operatorname{sgn} y=0, \quad \mu>0,
$$

which has a solution $S(\mu t), S$ being the generalized sine function, with zeros at $t=j \pi_{\alpha} / \mu, j \in \boldsymbol{Z}$. It is easily seen that, for any $N \in \boldsymbol{N}$, there is $\mu>0$ large enough so that $S(\mu t)$ has $N+1$ zeros in $[a, a+1]$. Then we compare (3.19) with $\left(\mathrm{H}_{\lambda}\right)$, where $\lambda>0$ is chosen such that $\lambda \min _{a \leq t \leq a+1} p(t) \geq \alpha \mu^{\alpha+1}$. By the Sturm 
comparison theorem for half-linear differential equations (Elbert 4]), we conclude that, for such values of $\lambda$, all solutions of $\left(\mathrm{H}_{\lambda}\right)$ have at least $N$ zeros in $[a, a+1]$, and, in particular, $x(t ; \lambda)$ has at least $N$ zeros in $[a, a+1]$. The proof of Lemma 3.1 is complete.

Proof of Theorem 1.2. We are now ready to prove Theorem 1.2. We make use of the generalized sine and cosine functions for the generalized Prüfer transformation, which consists in associating with a nontrivial solution $x(t ; \lambda)$ of $\left(\mathrm{H}_{\lambda}\right)$ the polar functions $\rho(t ; \lambda)$ and $\theta(t ; \lambda)$ defined by

$$
x(t ; \lambda)=\rho(t ; \lambda) S(\theta(t ; \lambda)), x^{\prime}(t ; \lambda)=\rho(t ; \lambda) C(\theta(t ; \lambda)) .
$$

It is easy to see that

$$
\rho(t ; \lambda)=\left(|x(t ; \lambda)|^{\alpha+1}+\left|x^{\prime}(t ; \lambda)\right|^{\alpha+1}\right)^{1 /(\alpha+1)}>0 .
$$

Moreover, it can be shown that $\theta=\theta(t ; \lambda)$ satisfies the first-order differential equation

$$
\theta^{\prime}=|C(\theta)|^{\alpha+1}+\frac{\lambda}{\alpha} p(t)|S(\theta)|^{\alpha+1} .
$$

Since $x(t ; \lambda)$ is increasing in $t \in[T, \infty)$, where $T$ is sufficiently large, the property (3.6) gives

$$
\frac{x^{\prime}(t ; \lambda)}{x(t ; \lambda)} \rightarrow 0 \quad \text { as } t \rightarrow \infty
$$

Then we have

$$
S(\theta(t ; \lambda))=\frac{x(t ; \lambda)}{\rho(t ; \lambda)}=\left(1+\left|\frac{x^{\prime}(t ; \lambda)}{x(t ; \lambda)}\right|^{\alpha+1}\right)^{-1 /(\alpha+1)} \rightarrow 1 \quad \text { as } t \rightarrow \infty
$$

and

$$
C(\theta(t ; \lambda))=\frac{x^{\prime}(t ; \lambda)}{\rho(t ; \lambda)}=\left(\left|\frac{x(t ; \lambda)}{x^{\prime}(t ; \lambda)}\right|^{\alpha+1}+1\right)^{-1 /(\alpha+1)} \rightarrow 0 \quad \text { as } t \rightarrow \infty .
$$

Thus we have

$$
\lim _{t \rightarrow \infty} \theta(t ; \lambda)=\frac{\pi_{\alpha}}{2}+2 m \pi_{\alpha} \quad \text { for some } m \in \boldsymbol{Z} .
$$

Then it is no loss of generality to suppose

$$
\lim _{t \rightarrow \infty} \theta(t ; \lambda)=\frac{\pi_{\alpha}}{2}
$$

The basic properties of $\theta(t ; \lambda)$ are as follows:

$\theta(t ; \lambda)$ is a continuous function of $\lambda \in(0, \infty)$ for each fixed $t \in[a, \infty)$; $\theta(t ; \lambda)$ is strictly increasing in $t \in[a, \infty)$ for each fixed $\lambda \in(0, \infty)$;

$\theta(t ; \lambda)$ is strictly decreasing in $\lambda \in(0, \infty)$ for each fixed $t \in[a, \infty)$.

The property (3.23) is an immediate consequence of Theorem 3.1 combined with the definition of $\theta(t ; \lambda)$. The property (3.24) is clear since, by (3.21), we have $\theta^{\prime}(t ; \lambda)>0(t \geq a)$ for each fixed $\lambda \in(0, \infty)$. To prove (3.25), assume the contrary. Then there exist $\lambda, \mu$ with $0<\lambda<\mu$ and $b \in[a, \infty)$ such that $\theta(b ; \lambda) \leq \theta(b ; \mu)$. 
Then a standard comparison theorem applied to the first-order scalar differential equation (3.21) implies that

$$
\theta(t ; \lambda) \leq \theta(t ; \mu) \quad \text { for } t \in[b, \infty) .
$$

Therefore, by (3.22) and (3.24), we have

$$
\frac{\pi_{\alpha}}{4}<\theta(t ; \lambda) \leq \theta(t ; \mu)<\frac{\pi_{\alpha}}{2}
$$

for all sufficiently large $t$. Since $T(\theta)=S(\theta) / C(\theta)$ is strictly increasing in $\theta \in$ $\left(\pi_{\alpha} / 4, \pi_{\alpha} / 2\right)$, this implies that

$$
\frac{x^{\prime}(t ; \lambda)}{x(t ; \lambda)}=\frac{1}{T(\theta(t ; \lambda))} \geq \frac{1}{T(\theta(t ; \mu))}=\frac{x^{\prime}(t ; \mu)}{x(t ; \mu)}
$$

for all sufficiently large $t$, which is a contradiction to (3.11). Thus (3.25) holds.

Since $\rho(t ; \lambda)>0(t \geq a)$, it follows from $(3.20)$ that $x(t ; \lambda)$ has a zero at $t=$ $c \in[a, \infty)$ if and only if there exists $j \in Z$ such that $\theta(c ; \lambda)=j \pi_{\alpha}$. Recall that $\theta(t ; \lambda) \rightarrow \pi_{\alpha} / 2$ as $t \rightarrow \infty$ for each $\lambda \in(0, \infty)$, and $\theta(a ; \lambda)$ is strictly decreasing in $\lambda \in(0, \infty)$. Then, by (3.7), (3.8) and (i) of Lemma 3.1, we find that

$$
\lim _{\lambda \rightarrow+0} \theta(a ; \lambda)=\frac{\pi_{\alpha}}{2} .
$$

Moreover, by (ii) of Lemma 3.1, we get

$$
\lim _{\lambda \rightarrow \infty} \theta(a ; \lambda)=-\infty
$$

Let us now consider $\theta(a ; \lambda)$ as a function of $\lambda \in(0, \infty)$. It is continuous and strictly decreasing and satisfies (3.26) and (3.27). Therefore, we can conclude that, for each $n=1,2, \cdots$, there exists $\lambda_{n}>0$ such that

$$
\theta\left(a ; \lambda_{n}\right)=-(n-1) \pi_{\alpha} .
$$

Then it is easily verified that the sequence $\left\{\lambda_{n}\right\}_{n=1}^{\infty}$ satisfies the properties (i), (ii) and (iii) of Theorem 1.2. The proof is complete.

\section{REFERENCES}

[1] E. A. Coddington and N. Levinson, Theory of Ordinary Differential Equations, McGraw-Hill, New York, 1955. MR 16:1022b

[2] W. A. Coppel, Stability and Asymptotic Behavior of Differential Equations, Heath, Boston, 1965. MR 32:7875

[3] M. Del Pino, M. Elgueta and R. Manasevich, Generalizing Hartman's oscillation result for $\left(\left|x^{\prime}\right|^{p-2} x^{\prime}\right)^{\prime}+c(t)|x|^{p-2} x=0, p>1$, Houston J. Math. 17 (1991), 63-70. MR 92e:34040

[4] Á. Elbert, A half-linear second order differential equation, Colloq. Math. Soc. J. Bolyai 30: Qualitative Theory of Differential Equations (Szeged) (1979), 153-180. MR 84g:34008

[5] Á. Elbert and T. Kusano, Oscillation and nonoscillation theorems for a class of second order quasilinear differential equations, Acta Math. Hungar. 56 (1990), 325-336. MR 93b:34039

[6] Á. Elbert, T. Kusano and M. Naito, On the number of zeros of nonoscillatory solutions to second-order half-linear differential equations, Ann. Univ. Sci. Budapest. Eötvös Sect. Math. 42 (1999), 101-131. MR 2001f:34056

[7] P. Hartman, Ordinary Differential Equations, John Wiley and Sons, New York, 1964. MR 30:1270

[8] H. Hoshino, R. Imabayashi, T. Kusano and T. Tanigawa, On second-order half-linear oscillations, Adv. Math. Sci. Appl. 8 (1998), 199-216. MR 99c:34059

[9] T. Kusano and M. Naito, A singular eigenvalue problem for the Sturm-Liouville equation, Differentsial'nye Uravneniya 34 (1998), 303-312; English transl., Differential Equations 34 (1998), 302-311. MR 99i:34039 
[10] T. Kusano and M. Naito, Sturm-Liouville eigenvalue problems for half-linear ordinary differential equations, Rocky Mountain J. Math. 31 (2001), 1039-1054.

[11] T. Kusano, M. Naito and T. Tanigawa, Second-order half-linear eigenvalue problems, Fukuoka University Science Reports 27 (1997), 1-7. MR 98f:34025

[12] T. Kusano and Y. Naito, Oscillation and nonoscillation criteria for second order quasilinear differential equations, Acta Math. Hungar. 76 (1997), 81-99. MR 98f:34071

[13] T. Kusano, Y. Naito and A. Ogata, Strong oscillation and nonoscillation of quasilinear differential equations of second order, Differential Equations and Dynamical Systems 2 (1994), 1-10. MR 97d:34030

[14] T. Kusano and N. Yoshida, Nonoscillation theorems for a class of quasilinear differential equations of second order, J. Math. Anal. Appl. 189 (1995), 115-127. MR 97f:34019

[15] H. J. Li and C. C. Yeh, Sturmian comparison theorem for half-linear second-order differential equations, Proc. Roy. Soc. Edinburgh 125A (1995), 1193-1204. MR 96i:34067

[16] J. D. Mirzov, On some analogs of Sturm's and Kneser's theorems for nonlinear systems, J. Math. Anal. Appl. 53 (1976), 418-425. MR 53:6005

[17] Z. Nehari, Oscillation criteria for second-order linear differential equations, Trans. Amer. Math. Soc. 85 (1957), 428-445. MR 19:415a

Department of Applied Mathematics, Faculty of Science, Fukuoka University, FukuOKA 814-0180, JAPAN

E-mail address: tkusano@cis.fukuoka-u.ac.jp

Department of Mathematical Sciences, Faculty of Science, Ehime University, MatSUYAMA 790-8577, JAPAN

E-mail address: mnaito@math.sci.ehime-u.ac.jp 